\title{
The Effect of Spectral Power Distribution of White Light-Emitting Diodes on Eye Fatigue
}

\author{
Junya SUGIMOTO*, Yukio AKASHI*,†, Yuki HIRAGA*, Masaru INATANI**, Emi KAGA*** and \\ Shota SHIMONISHI***
}

\author{
* Graduate School of Engineering, University of Fukui \\ ** Department of Ophthalmology, Faculty of Medical Sciences, University of Fukui \\ *** Toyoda Gosei Co., Ltd.
}

Received January 21, 2021, Accepted May 17, 2021

\begin{abstract}
This study investigates the effect of blue light emitted by phosphor-converted white light-emitting diodes (LEDs) on people's eye fatigue. We conducted an experiment in which participants performed a visual task under lighting conditions using a white LED (B-LED) in which blue light excites phosphors and white LEDs (V-LEDs) in which violet light excites two types of phosphors. One of the two V-LEDs had a spectral power distribution mimicking those of the sun (S-LED). During the experiment, we measured participants' subjective eye fatigue, number of eye blinks, accommodation power and critical flicker frequency. The results of the subjective evaluation suggested that participants' eye fatigue under the B-LED lighting was worse than under the S-LED or V-LED lighting. The results of the eyeblink and accommodation measurements suggested that participants' eye fatigue under the B-LED lighting was worse than under the S-LED lighting. In the range of the white LEDs employed in our experiment, we found that participants' eye fatigue under lighting conditions with a blue-light-excited white LED could be worse than under a violetlight-excited white LED. The results of the objective response measurements suggested that the fatigue of eye muscles is the principal factor causing subjective eye fatigue.
\end{abstract}

KEYWORDS: eye fatigue, white LED, physiological index, subjective evaluation, spectral power distribution

\section{Introduction}

Recently, phosphor-converted white light-emitting diodes (LEDs) have been widely used as light sources for general lighting. The white LEDs have higher lamp efficiency, lower heat generation and longer life over conventional light sources. In these LEDs, blue light (or violet light) excites the phosphor to emit white light. It is relatively easy for LED manufacturers to alter the spectral power distributions (SPD) of LEDs by changing phosphor types and amounts. Recent studies have experimented under different SPD conditions investigating the effect of blue light on eye fatigue ${ }^{1-6)}$.

Wang et al. evaluated the eye fatigue of participants who used visual display terminals (VDTs) under lighting conditions using a white LED and a fluorescent lamp ${ }^{1}$. The results of subjective and objective response measurements revealed that white LED lighting, which emits more blue-light radiation, causes worse eye fatigue than fluorescent lighting. Zhang et al. conducted an experiment in which participants performed a VDT task under lighting conditions with two types of white LEDs $^{2)}$. One white LED contained more radiation in the blue-light wavelength range than the other one. The results suggested that the white LED containing more blue light causes worse eye fatigue.

However, the two white LEDs had different correlated colour temperatures (CCTs) of 6,395 K and 2,850 K in Zhang et al.'s experiment. Therefore, the participants could recognise under which lighting they worked during the experiment. Further, each white LED consisted of a white, red, green and blue LED. Such an SPD differs from the white LEDs widely used for recent general illumination. Additionally, in Wang et al. and Zang et al.'s experiments, white LEDs illuminated the perimeter areas surrounding the computer displays. During the experiment, the participants who mainly gazed at the displays could be little affected by the SPD of the white LEDs.

We investigated the effect of light sources containing blue light on eye fatigue by using a blue-light-excited white LED and two violet-light-excited white LEDs, which are used for general lighting. The blue-lightexcited LED excites phosphors with blue light and emits white light, whereas the two violet-light-excited

\footnotetext{
${ }^{\dagger}$ Corresponding Author: Yukio Akashi akashi@u-fukui.ac.jp
} 
LEDs excite phosphors with violet light and emits white light. The two types of violet-light-excited LEDs had different phosphor types. In our experiment, the three white LEDs had CCTs of $\sim 5,000 \mathrm{~K}$ so that participants could not identify the types of light sources from the obvious colours. During the experiment, the participants performed a visual task with visual stimuli printed on white paper illuminated by white LEDs because the participants' eyes could receive more light from the test light sources while performing the visual task printed on white paper than the VDT task.

\section{Objective indexes of eye fatigue}

Previous studies evaluated the degree of eye fatigue by comparing the results of objective response measurements and subjective evaluations ${ }^{1-14}$. Table 1 summarises the results of objective response measurements and subjective evaluations of these studies.

The ++ mark in the objective response column in Table 1 shows that the objective response could be reliable to evaluate the degree of eye fatigue. The criteria for the reliability to which we referred are discussed below.

(1) A significant difference in objective response measurements between the before and after visual tasks or among lighting conditions

(2) A significant difference in subjective eye-fatigue evaluations between the before and after visual tasks or among lighting conditions

(3) Consistency between the results of the objective response measurements (1) and eye-fatigue evaluations (2).

The + mark in Table 1 shows a significant difference in the measured value of the objective response between the before and after visual tasks or among different lighting conditions. However, there was no significant difference among the lighting conditions in subjective eye-fatigue evaluations.

The $\mathrm{x}$ mark in Table 1 shows that there was no significant difference in the measured value of the objective response between the before and after visual tasks or among illumination conditions. There was no significant difference in subjective eye-fatigue evaluations among the lighting conditions.

Table 1 shows that critical flicker frequency $(\mathrm{CFF})$, eye blink and accommodation power have relatively large numbers of ++ and + marks and, therefore, these objective indices are useful to evaluate the state of eye fatigue. CFF reflects fatigue originating in the cerebral cortex ${ }^{15)}$. Eyeblink represents the visual fatigue of the extraocular muscles because of eye movements ${ }^{16)}$, and accommodation power reflects the visual fatigue of the ciliary muscles' accommodative system ${ }^{17}$. Kubo et al. stated that eye fatigue claimed by workers might originate in the cerebral cortex and ocular muscle fatigue, often occurring in combination ${ }^{4}$. From the above described survey, we selected CFF, eyeblink and accommodation power to measure the state of fatigue related to the cerebral cortex and ocular muscle.

Table 1 Objective responses used for evaluating eye fatigue.

\begin{tabular}{|c|c|c|c|c|c|c|c|c|c|c|c|c|c|c|c||c|}
\hline & & & & & & & & \\
\hline \\
\hline
\end{tabular}




\section{Experiment}

The experiment was carried out in our laboratory. Participants sat in front of a desk and performed a visual task for $45 \mathrm{~min}$. For the visual task, we used Landolt rings printed on white paper. We monitored any change in the state of eye fatigue during the visual task by dividing the 45 -min visual task into three 15 -min sessions. We measured the state of eye fatigue using the accuracy rate of the visual task, the subjective evaluation, the number of eyeblinks, CFF and accommodation power under three white LED lighting conditions. The participants daily performed the visual task under one of three lighting conditions with different white LEDs. The participants completed the visual task under three LED conditions in three days and started the visual task at the same time each day. This experimental plan was approved by the Research Ethics Committee of the University of Fukui. Before participating in the experiment, all participants signed informed consent forms.

\subsection{Participants}

In the experiment, 24 students (18 males and six females, 21-24 years old) of the University of Fukui participated. Participants who needed visual correction wore glasses or contact lenses that they use for normal visual tasks. All participants had visual acuity of 0.7 or higher when participating in the experiment.

\subsection{Experiment period}

The experimental period was between 10:30 AM and 6:00 PM. Each participant selected one from four experimental periods: (1) 10:30-12:00, (2) 13:00-14:30, (3) 14:45-16:15 and (4) 16:30-18:00. We selected these four experiment periods in which participants' activity is high, eliminating early morning hours in which participants are sleepy or tired. Additionally, each participant has biological rhythms that repeat arousal and sleep, which change depending on the time of day. Therefore, each participant started the visual task at the same time each day.

\subsection{Experimental laboratory}

We experimented in a laboratory with a width of $4.5 \mathrm{~m}$, depth of $6 \mathrm{~m}$ and a ceiling height of $2.7 \mathrm{~m}$. The reflectances of the interior surfaces were $90 \%$ for the ceiling, $60 \%$ for the walls and $10 \%$ for the floor. All openings in the laboratory were covered with a shading curtain with zero percent transmittance to shut out daylight. All the lighting fixtures installed on the ceiling were turned off, and a lighting fixture of an experimental setup described below illuminated the work surface.

\subsection{Experimental setup}

Figure 1 shows the experimental setup, consisting of a metal frame stand, a lighting fixture, a table, a parti-

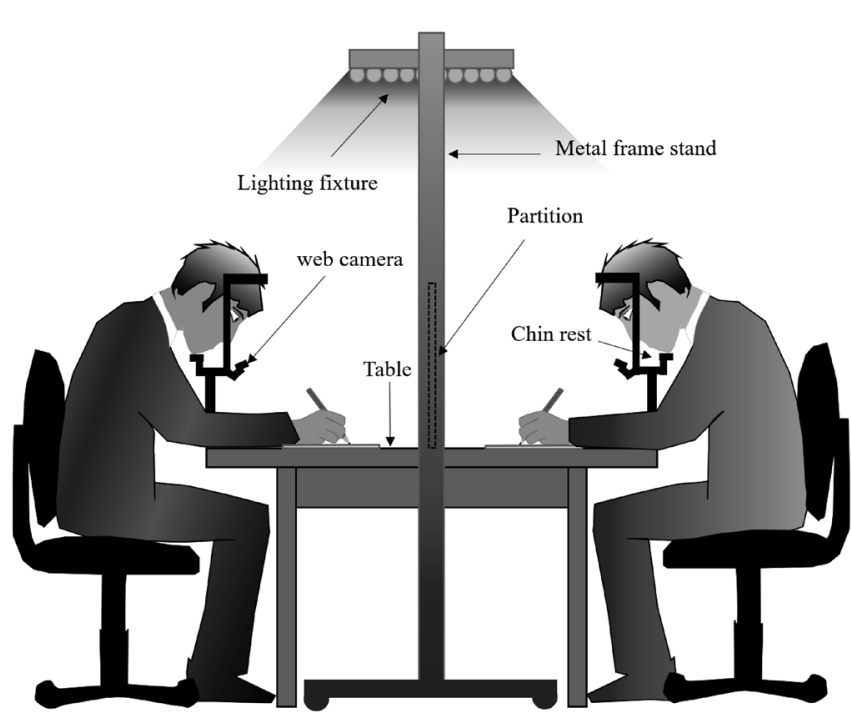

Figure 1 Experimental setup.

tion, a web camera and a chin rest.

We prepared three lighting fixtures with different SPDs. Each lighting fixture was suspended from the top of the metal frame stand, which could be easily replaced with the others. Each lighting fixture was a stainlesssteel box with a width of $600 \mathrm{~mm}$, a depth of $632 \mathrm{~mm}$ and a height of $46 \mathrm{~mm}$, containing 15 white LED tubes $600 \mathrm{~mm}$ long. A translucent cover filtered each white LED tube consisting of 120 LED chips. Each lighting fixture had a rotary knob that could simultaneously control the 15 white LED tubes. By rotating the rotary knob, an experimenter dimmed the LEDs to change the illuminance on the work surface.

The table was $1,200 \mathrm{~mm}$ wide, $900 \mathrm{~mm}$ deep and $720 \mathrm{~mm}$ high. On the $900 \mathrm{~mm}$ sides of the table, two participants sat face-to-face. Two participants at a time participated in the experiment. However, a $355 \mathrm{~mm}$ high and $680 \mathrm{~mm}$ wide translucent partition divided the table into two so that the two participants could not view each's visual tasks. A sheet of grey cloth with a reflectance of $\sim 26 \%$ covered the tabletop.

\subsection{Visual task}

The visual task used in the experiment was a search task. The target of the search task was a set of 1,600 Landolt rings printed with achromatic ink in the centre of A4 paper arranged 40 lengthwise and widthwise. The 1,600 Landolt rings were aligned in a square with sides of $60 \mathrm{~mm}$. Each Landolt ring had a diameter of $1.5 \mathrm{~mm}$ with a gap in any one of the eight orientations out of the top, top-right, right, bottom-right, bottom, bottomleft, left and top-left, of which 200 in each of the eight directions had gaps. These 1,600 Landolt rings in eight orientations were randomly placed. Figure 2 shows a sample A4 paper with the Landolt rings printed on it.

The subjects' visual task in the experiment was to search for Landolt rings with a certain gap orientation. 


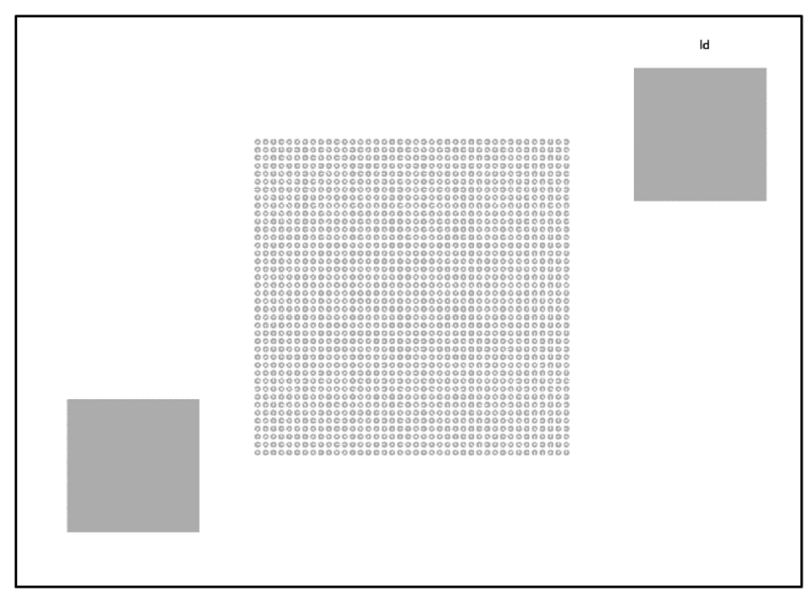

Figure 2 Visual target used in the experiment.

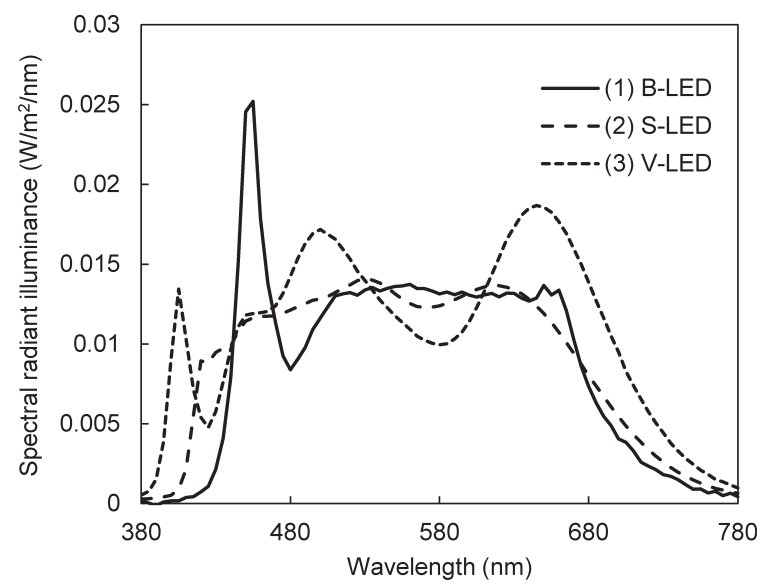

Figure 3 Spectral radiant illuminance of the three light sources to illuminate the desk at $1000 \mathrm{~lx}$ in the experiment.

Before the experiment, an experimenter selected one of the eight directions of the Landolt ring gap in random order and let each subject know the specified gap orientation. As soon as hearing the experimenter's signal, the subject started searching for the Landolt rings with the specified gap orientation and marked them with a pencil. The grey squares with $3 \mathrm{~cm}$ sides on the upper-right and lower-left corners were printed with the same ink as the Landolt rings. Therefore, the two grey squares had the same reflectance as the Landolt rings, ensuring a size large enough to be measured by a luminance metre. We used the two grey squares to measure the luminance of the Landolt rings. The luminance (Weber) contrast of the Landolt rings against the background was constant at 0.33 .

\subsection{Independent variable}

The independent variable in this study was SPD of white LEDs. Figure 3 shows the three levels of SPDs used in the experiment.

(1) A blue-light-excited white LED in which blue light excites red and green phosphors (blue-excited
LED in the body of this paper or B-LED in figures and tables)

(2) A violet-excited white LED in which violet light excites red, green and blue phosphors to make the SPD closer to that of the sun (solar LED or S-LED)

(3) A violet-excited LED in which violet light excited various phosphors (violet-excited LED or V-LED)

Figure 3 shows that the SPDs of the three white LEDs differed from each other. The CCTs of the three light sources were set to $\sim 5,000 \mathrm{~K}$. The actual CCTs of (1), (2) and (3) were 5,228 K, 5,140 K and 5,056 K, respectively. We selected similar CCTs for the test LEDs so that the participants could not recognise under which LED lighting conditions they performed the visual task during the experiment. The horizontal illuminance on the tabletop for any of the three LEDs was fixed at $1,000 \mathrm{~lx}$. Therefore, the participants could see the targets clearly under any experimental condition. The averaged vertical illuminance at the participants' eyes was $190 \mathrm{~lx}$. The background luminance of the visual target was $230 \mathrm{~cd} / \mathrm{m}^{2}$.

\subsection{Dependent variables}

\subsubsection{Accuracy rate of visual task}

We used the accuracy rate $\left(A_{\mathrm{r}}\right)$ as one of the dependent variables. The $A_{\mathrm{r}}$ is the ratio of the number of Landolt rings correctly detected by a participant for $15 \mathrm{~min}\left(N_{\mathrm{c}}\right)$ against the number of Landolt rings seen by the same participant for $15 \mathrm{~min}\left(N_{\mathrm{s}}\right)$. The $A_{\mathrm{r}}$ was obtained using Eq. (1) for each of the three task sessions under each of the three SPD conditions for each of the 24 participants. We averaged the $A_{\mathrm{r}}$ values under each of the three SPD conditions over the 24 participants. The $A_{\mathrm{r}}$ was used as a dependent variable because participants can claim the number of Landolt rings seen, even if they do not focus on the searching task because of eye fatigue. However, it is challenging for participants to improve the accuracy of detection when they are tired.

$$
A_{\mathrm{r}}=\left(N_{\mathrm{c}} / N_{\mathrm{s}}\right) \times 100(\%)
$$

\subsubsection{Measuring subjective evaluation}

Table 2 shows eight statements from (1) to (8) used for participants to describe the visual conditions as dependent variables. We selected the eight statements from the eye-fatigue descriptions of the survey of subjective symptoms ${ }^{18)}$ provided by the Japan Society for Occupational Health. We asked participants the appropriateness of each statement to describe the state of their visual conditions. The participants used a sevenpoint response scale according to descriptors attached to the even-numbered points, which were 0 (not at all), 2 (a little), 4 (rather) and 6 (very much). The participants completed a subjective evaluation before the first visual 
Table 2 Statements used in subjective evaluation.

\begin{tabular}{|l||l|}
\hline & Statements describing visual condition \\
\hline \hline$(1)$ & It is hard to open my eyes \\
\hline$(2)$ & Characters appear blurred to me \\
\hline$(3)$ & I feel like I have poor eyesight \\
\hline$(4)$ & My eyes feel hot \\
\hline$(5)$ & I have eye fatigue \\
\hline$(6)$ & I have eye pain \\
\hline$(7)$ & My eyes feel dry \\
\hline$(8)$ & The task causes glare to me \\
\hline
\end{tabular}

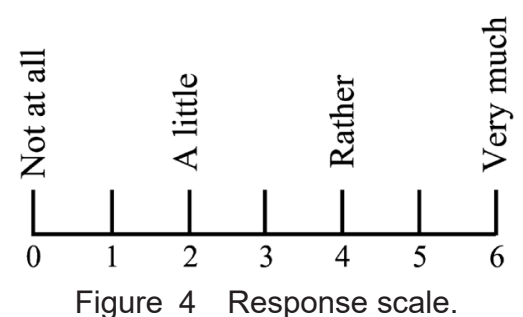

task. We treated the first value as the reference value. The participants repeated the same subjective evaluation after each of the three 15-min visual task sessions. Consequently, we obtained four subjective evaluations from each participant, i.e., the reference and the three post-task evaluations.

\subsubsection{Measuring Eyeblinks}

An eyeblink is an instantaneous closing of both eyelids while the person is awake. Tada et al. reported that frequent eye blinking is a sign of eye fatigue ${ }^{16)}$. Therefore, we regarded an increase in the number of eyeblinks as an increase in the degree of eye fatigue.

We used a web camera (HD webcam C615, Logitech) to count the number of eyeblinks. We placed a web camera on the chin rest (Figure 1) and connected it to a personal computer. We continuously videotaped participants' eyes during the experimental sessions. After the experiment, we watched the video recordings of the participants' eye images and counted the number of eyeblinks for each minute of the 15-min task periods.

\subsubsection{Measuring critical flicker frequency}

CFF is the threshold-flickering frequency at which an observer can start recognising light-flickering. The lower the frequency of the flickering light, the easier it is to perceive it. Nishimura et al. found that people had lower CFFs with increased eye fatigue but higher CFFs without eye fatigue ${ }^{19)}$.

In the CFF measurement, we measured two $\mathrm{CFF}$ values using a CFF measurement device (Type II
T.K.K.501c, Takei Scientific Instruments Co., Ltd.). The one $\mathrm{CFF}$ value measured the frequency of flickering light while it increased from a low $(20 \mathrm{~Hz})$ to a high frequency $(60 \mathrm{~Hz})$, and the other $\mathrm{CFF}$ value measured while it decreased from a high $(60 \mathrm{~Hz})$ to a low frequency $(20 \mathrm{~Hz})$. In the ascending series, participants identified the threshold frequency between the state at which the light-flickering was recognisable and where the light-flickering was unrecognisable. In the descending series, participants identified the threshold frequency between the state at which the light-flickering was unrecognisable and at which the light-flickering was recognisable.

The participants selected the reference CFF value before performing the visual task and a post-task CFF value after each visual task session $(15 \mathrm{~min})$ out of the three repeated sessions. In each $\mathrm{CFF}$ measurement, we obtained six CFF values from a participant under each of the three SPD conditions. Finally, we averaged the six $\mathrm{CFF}$ values to obtain a single $\mathrm{CFF}$ value for the participant under each of the three conditions.

Takeda et al. $^{20)}$ reported that participants needed to practice the $\mathrm{CFF}$ measurement before the real experiment. An unpracticed participant tends to select lower $\mathrm{CFF}$ values. As participants become used to the CFF measurement, they tend to select higher $\mathrm{CFF}$ values. As an inexperienced participant repeats the CFF measurement during the experiment, the participant's skill will increase, and the measured CFF value will increase. Such a tendency could mask a potential reduction in CFF because of eye fatigue. This measurement flaw was prevented by allowing all participants to practice the CFF measurement sufficiently at least one day before the first experiment day.

\subsubsection{Measuring accommodation function}

When a person observes an object at a constantly short distance, the refractive index of the crystalline lens swings rhythmically, called accommodative microfluctuations. The accommodative micro-fluctuation can be characterised by two principal regions of dominant activity, namely a low-frequency region with frequencies lower than $0.6 \mathrm{~Hz}$ and a high-frequency region (High Frequency Component: HFC) with frequencies between $1.0 \mathrm{~Hz}$ and $2.3 \mathrm{~Hz}^{21}$. High frequency fluctuation occurs because of the quiver of the ciliary muscle and increases with excessive load on the ciliary muscle. In a healthy ciliary muscle, even when the load on the ciliary muscle increases slightly, HFC does not increase. However, in the tired ciliary muscle, the HFC increases with a small accommodative load ${ }^{17}$. Therefore, researchers have used the HFC to evaluate the state of subjects' visual fatigue ${ }^{22,23)}$.

We obtained an HFC value by integrating common logarithms of frequency spectra $(\mathrm{dB})$ obtained by ap- 


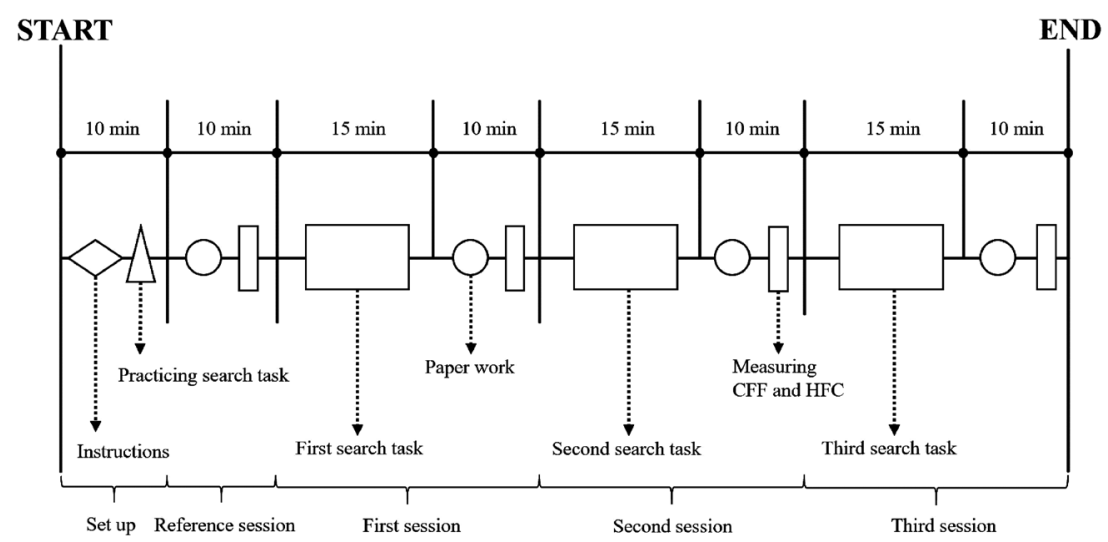

Figure 5 Experimental procedure for each of the three SPD conditions.

plying a Fast Fourier Transform to accommodative micro-fluctuations in the high-frequency region $(1.0 \mathrm{~Hz}$ and $2.3 \mathrm{~Hz})^{17)}$. The HFC is a relative value with no units. Given the above, we used the HFC as an index representing the degree of eye fatigue in our experiment. We measured the HFC using an accommodation function analyser (auto refractometer AR-1, NIDEK) and software to analyse accommodation power (AA-2, NIDEK).

\subsection{Experimental procedure}

We conducted the experiment by following the procedure from (1) to (7) listed below. Figure 5 depicts the experimental procedure for each of the three SPD conditions. We used only one flicker measurement device and one accommodation measurement device. We required two participants at a time to participate in the experiment to improve efficiency. However, when a participant measures $\mathrm{CFF}$ or $\mathrm{HFC}$, the fatigue of the other participant could change. Therefore, we delayed the starting time of the visual task for the second subject by $1 \mathrm{~min}$. We counterbalanced the presentation order of the light sources over the 24 subjects.

(1) Two participants were escorted to a laboratory where one condition from the three SPD conditions was selected in advance. The participants received instructions on the experimental procedure. The participants placed their chins on the chinrests and practiced the search task. During the practice, the participants adapted to the luminance of the work surface. (10 min)

(2) The participants made subjective evaluations as a reference before conducting the visual task. The experimenter measured the participants' $\mathrm{CFF}$ and $\mathrm{HFC}$ as the reference values. (10 min)

(3) One of the two participants started performing the search task for 15 min under the given lighting condition. The other participant started the search task 1 min later.

(4) The first participant made a subjective evaluation. The experimenter measured the $\mathrm{CFF}$ and $\mathrm{HFC}$

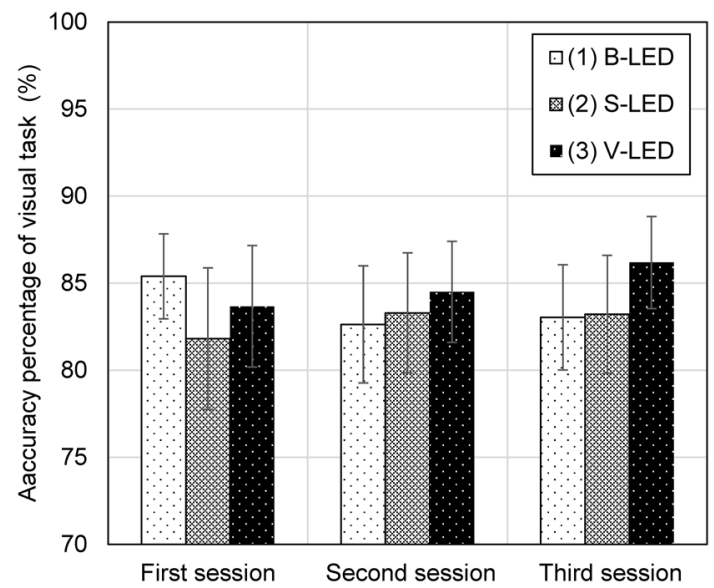

Figure 6 Accuracy percentage (\%) and standard error of visual task.

of the first participant. The second participant followed the procedure of the first participant. (10 min)

(5) We repeated the procedure from (3) to (4) twice (three times in total). We completed the experiment under the first SPD condition. Therefore, the time for the visual task was $45 \mathrm{~min}$.

(6) We repeated the procedure from (1) to (5) but under the second SPD condition at the same time on the second day.

(7) We repeated the procedure from (1) to (5) but for the third SPD condition at the same time on the third day.

\section{Results}

We compared the degree of eye fatigue among different SPD conditions by analysing the experimental data regarding the accuracy rate of the visual task, subjective evaluation, eye blinking, $\mathrm{CFF}$ and $\mathrm{HFC}$ obtained from the 24 participants.

\subsection{The accuracy rate of the visual task}

Figure 6 compares the accuracy rates among the three SPD conditions for each of the three 15-min vi- 
sual task sessions. We applied a two-way analysis of variance (ANOVA) and post-hoc analyses (Tukey HSD method) to the experimental data and compared the accuracy rates among the three SPD conditions. The results of the analyses showed no major effects of light source on the Landolt ring detection accuracy.

\subsection{Subjective evaluation}

We analysed the data of the subjective evaluation by scaling the degree of appropriateness of each statement, describing the state of the participant's visual conditions by using the descriptors attached to the even-numbered points, namely 0 (not at all), 2 (a little), 4 (rather) and 6 (very much).

However, the subjective evaluation of an individual participant could change because of daily fluctuations in the participant's physical and mental conditions. Since each participant performed the visual task over three days, we eliminated daily fluctuations in physical and mental conditions on the subjective evaluation. Therefore, we analysed the experimental data appropriately by investigating the amount of change in the subjective evaluation by subtracting the reference value from the post-task value after each of the three visual task sessions. We then took the average of the changes in the subjective evaluation for the three visual task sessions under each SPD condition.

We applied a two-way ANOVA and Tukey HSD method to the experimental data, the differences in the subjective evaluation from the reference values, and the post-task for all 24 participants. In this subclause, we report predominantly on the statements that showed statistical significance. Figures 7, 8 and 9 show the results of the average differences in subjective evaluations among three SPD conditions for the statements in which we found statistical significance.

The results of the statistical analyses showed that statements where the SPD condition had the most effects were Statement (2): Characters appear blurred to me $(\phi<0.05)$ and Statement (5): I have eye fatigue $(\phi<0.01)$. Additionally, the results of the post-hoc analyses among the three SPD conditions suggested the followings.

In Figure 7, showing the results of Statement (2): Characters appear blurred to me, we found that the solar LED had a significantly higher increment in the subjective evaluation than the blue-excited LED $(p<0.05)$. Therefore, we found that characters in the visual task under the solar LED were blurrier than under the blueexcited LED.

In Figure 8, showing the results of Statement (5): I have eye fatigue, the blue-excited LED had a significantly larger increment in the subjective evaluation than the solar LED $(p<0.01)$. Further, the blue-excited LED had a significantly larger increment in subjective evaluation than the violet-excited LED $(p<0.05)$. There-

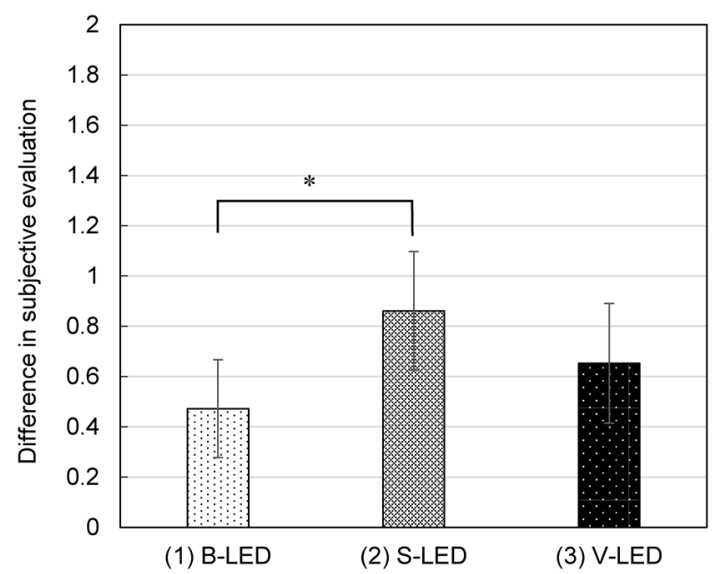

Figure 7 Difference in subjective evaluation and standard error for Statement (2): Characters appear blurred to me $(*: p<0.05)$.

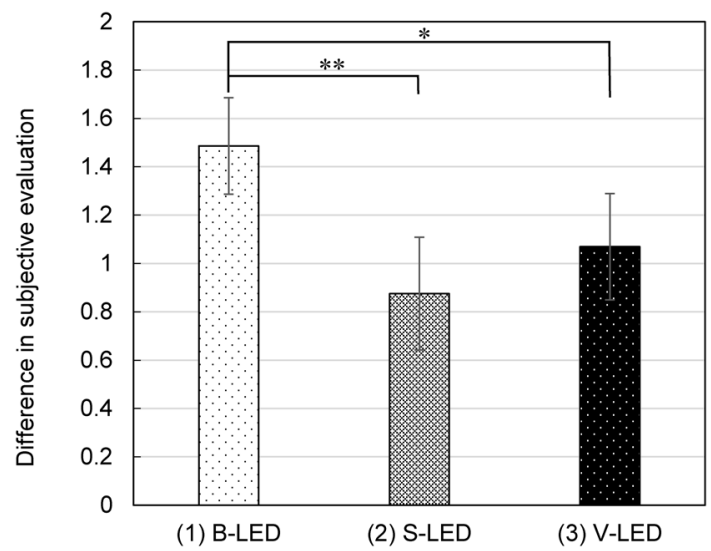

Figure 8 Difference in subjective evaluation and standard error for Statement (5): I have eye fatigue $(*: p<0.05$, $* *: p<0.01)$.

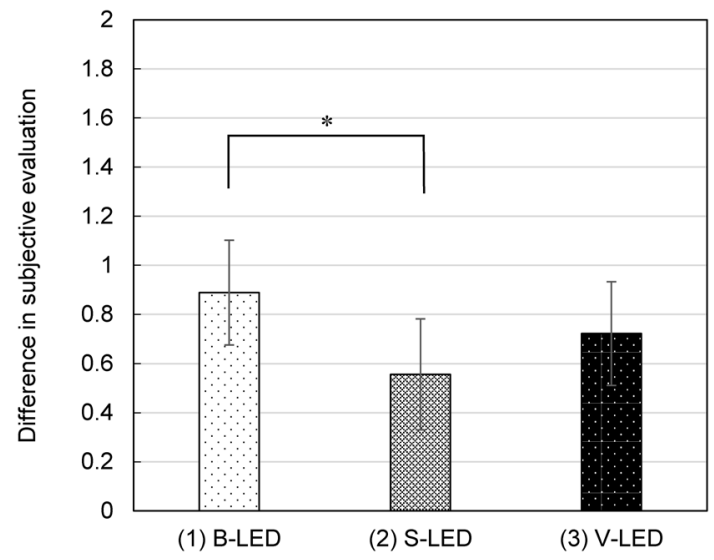

Figure 9 Difference in subjective evaluation and standard error for Statement (6): I have eye pain (*: $p<0.05)$.

fore, the participants under the blue-excited LED were more fatigued than under the solar LED and violetexcited LED.

In Figure 9, showing the results of Statement (6): 


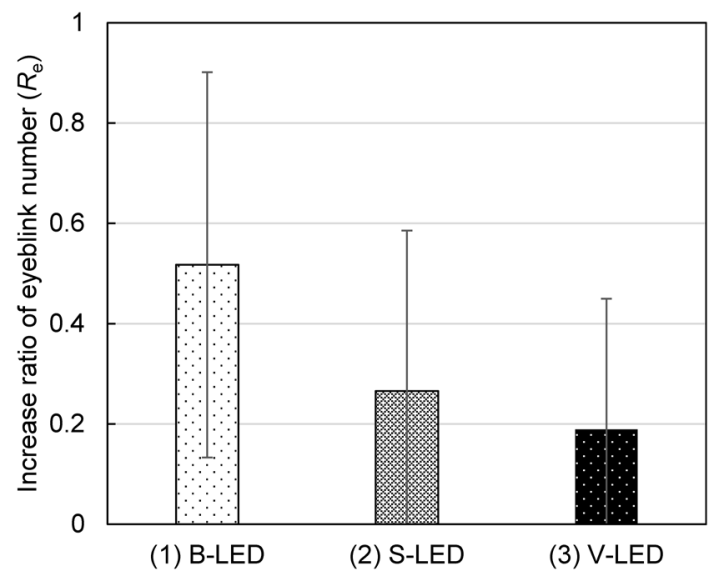

Figure 10 Increase ratio $\left(R_{\mathrm{e}}\right)$ and standard error of eyeblink number. $R_{\mathrm{e}}=\left(N_{2}-N_{1}\right) / N_{1}$ where $N_{1}$ is eyeblink number in the first half period and $N_{2}$ is that in the second half period.

I have eye pain, the blue-excited LED had a significantly higher increment in the subjective evaluation than the solar LED $(\phi<0.05)$. Therefore, the participants under the blue-excited LED had worse eye pain than under the solar LED.

\subsection{Eyeblinks}

In the experiment, we measured the number of eyeblinks every minute during the 15 -min visual task. We investigated the increases in eyeblink caused by eye fatigue during the visual task by calculating the increase of the number of eyeblinks. We first divided the 15-min visual task period into a first and second half. Then, we calculated the increase ratio $\left(R_{\mathrm{e}}\right)$ of eyeblink number between the second half $\left(N_{2}\right)$ and the first half $\left(N_{1}\right)$ over the eyeblink number in the first half period $\left(N_{1}\right)$ as shown in Eq. (2).

$$
R_{\mathrm{e}}=\left(N_{2}-N_{1}\right) / N_{1}
$$

We applied a two-way ANOVA and Tukey HSD method to the ratios of eyeblinks obtained from 24 participants under the three SPD conditions. However, the results of these statistical analyses showed no significant difference among the three SPD conditions.

\subsection{Results of change in CFF measurements}

We obtained four CFF values, namely the reference and three post-task values under each of the three SPD conditions from each of the 24 participants during the experiment. However, the measured CFF values could change because of daily fluctuations in the participants' physical conditions. Therefore, before analysing the experimental data, we first calculated the difference in $\mathrm{CFF}$ value by subtracting the reference $\mathrm{CFF}$ value $\left(C_{\mathrm{r}}\right)$ from each of the three post-task $\mathrm{CFF}$ values $\left(C_{\mathrm{p}}\right)$. Second, we calculated the change rate $\left(R_{\mathrm{c}}\right)$ by dividing

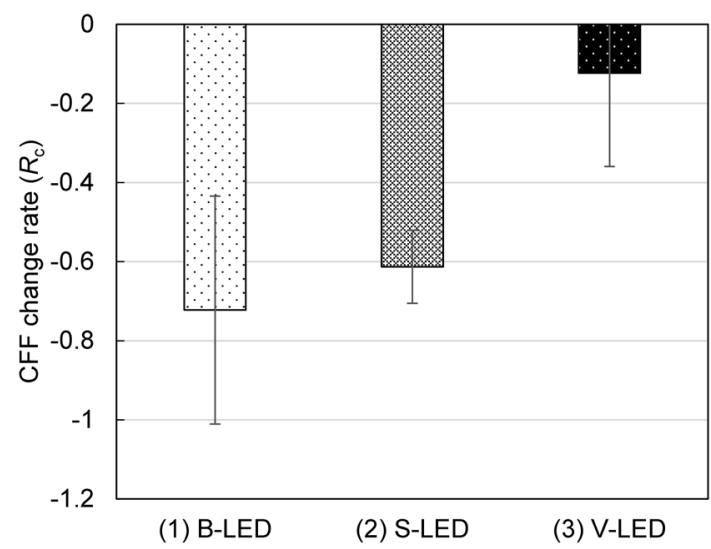

Figure $11 \mathrm{CFF}$ change rate $\left(R_{\mathrm{C}}\right)$ and standard error. $R_{\mathrm{c}}=\left(C_{\mathrm{p}}-C_{\mathrm{r}}\right) / C_{\mathrm{r}}$ where $C_{\mathrm{r}}$ is the reference CFF value and $C_{p}$ is the post-task CFF value $\left(C_{p}\right)$.

the difference in $\mathrm{CFF}$ value by the reference $\mathrm{CFF}$ value $\left(C_{\mathrm{r}}\right)$ as shown in Eq. (3).

We took the average of the three change rates over the three visual task sessions under each of the three SPD conditions. Figure 11 shows the CFF change rates under the three SPD conditions. Because the CFF value decreases as a person becomes tired, the CFF change rate has a negative value.

$$
R_{\mathrm{c}}=\left(C_{\mathrm{p}}-C_{\mathrm{r}}\right) / C_{\mathrm{r}}
$$

We applied a two-way ANOVA and Tukey HSD method to the CFF change rates for all 24 participants. However, the results of these statistical analysis showed no significant difference among the three SPD conditions.

\subsection{Results of accommodation power (HFC) mea- surements}

We obtained four HFC values, namely the reference and three post-task values, under each of the three SPD conditions for each of the 24 participants. We calculated the ratio $\left(R_{\mathrm{h}}\right)$ of each post-task HFC value $\left(H_{\mathrm{p}}\right)$ to the reference HFC value $\left(H_{\mathrm{r}}\right)$ under the three SPD conditions for each of the 24 participants as shown in Eq. (4). We called this ratio the HFC ratio. Then, we took the average over the three post-task HCF values and the 24 participants. Figure 12 compares the HFC ratios under the three SPD conditions.

$$
R_{\mathrm{h}}=H_{\mathrm{p}} / H_{\mathrm{r}}
$$

We applied a one-way ANOVA and Tukey HSD method to the HFC ratios obtained from 24 participants under the three SPD conditions. The results of the oneway ANOVA showed a significant effect $(p<0.05)$ on the SPD. Additionally, the results of the post-hoc analyses revealed that the blue-excited LED had a significantly larger HCF ratio than the solar LED $(p<0.05)$ (Figure 12). 


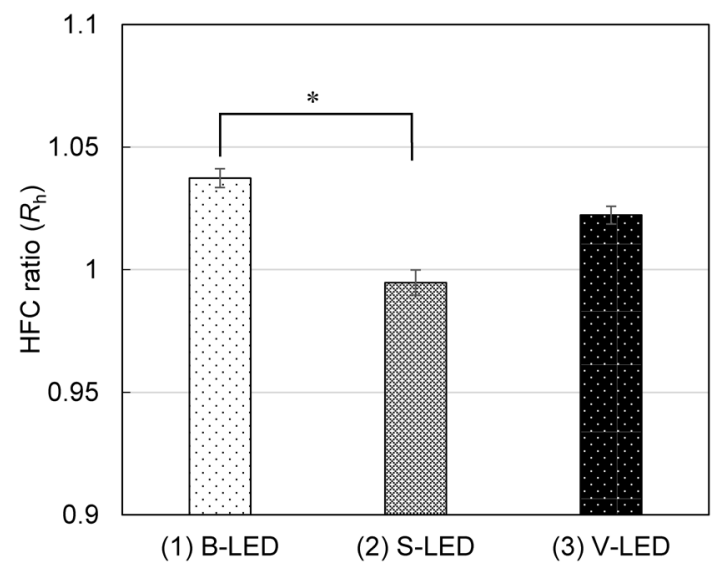

Figure $12 \mathrm{HFC}$ ratio $\left(R_{\mathrm{h}}\right)$ and standard error. $R_{\mathrm{h}}=H_{\mathrm{p}} / H_{\mathrm{r}}$ where $H_{\mathrm{p}}$ is the post-task HFC value and $H_{\mathrm{r}}$ is the reference HFC value $(*: p<0.05)$.

\section{Discussion}

\subsection{Task performance}

From the accuracy rates of the visual task in Figure 6 , no significant difference exists among the three SPD conditions. Figure 6 also shows that the accuracy rates of the three SPD conditions were maintained at elevated levels between $83 \%$ and $87 \%$. These consistently high accuracy rates show that the visual task exerted a constant load on the visual and cognitive functions of the participants during the experiment and that participants could keep concentrating on the visual task.

\subsection{Subjective evaluation}

The results of the subjective evaluation suggested that the participants under the blue-excited LED lighting became more visually fatigued after the visual task than under the solar LED and violet-excited LED lighting (Statements (5): I have eye fatigue and Statement (6): I have eye pain). However, visual targets under the solar LED lighting appeared blurrier than those under the blue-excited LED lighting. In this experiment, we used an illuminance of 1,000 lx on the tabletop. We conclude that the participants had no difficulty viewing the visual targets at such a high illuminance; therefore, the participants did not reflect the states of eye fatigue in response to Statement (2): characters appear blurry. Instead, the participants only referred to the appearance of the visual targets. However, no evidence supports the hypothesis that the blurriness of visual targets might not affect the state of eye fatigue. Further research should investigate why visual targets under solar LED lighting appeared blurrier than those under the blue-excited LED lighting.

\subsection{Objective responses}

The results of the subjective eye-fatigue evaluation showed a significant difference between the blue-excited LED and the solar LED. It is also shown that the in- crease in the degree of eye pain under the blue-excited LED was higher than under the solar LED.

Among the objective responses, the results of the HFC ratio showed that the blue-excited LED had a significantly larger HFC ratio than the solar LED. Since the increase in $\mathrm{HFC}$ reflects the fatigue of the ciliary muscle, the results of subjective evaluation, eyeblink and accommodation function consistently support that participants under the blue-excited LED became more visually tired during the 45-min visual task than under the solar LED. These results of both the subjective evaluations and the objective responses matched each other well.

\subsection{Relationship between spectral characteristics of light source and eye fatigue}

The intensity of a light-emitting stimulus causing a human sensation, perception or physiological reaction depends on both the spectral radiant power of the light and the spectral sensitivity of a photoreceptor receiving the light from the stimulus. It is critical to clarify which photoreceptor mediates the mechanisms from the input stimulus to the output response based on the correspondence between the spectral radiant energy of light and human sensation, perception or physiological response. If the mechanisms could be found, it would be possible for us to tune the SPD of the white LED to be less stimulating to the photoreceptor.

This experiment showed a tendency that the blueexcited white LED caused worse eye fatigue than the violet-excited white LEDs. Therefore, photoreceptors that have peaks of sensitivity at a shorter wavelength range than the spectral sensitivity of cones could be related to eye fatigue.

Therefore, we first analysed our experimental data using the blue-light hazard function described in standards such as IEC $62471^{24)}$ and IEC/TR $62778^{25)}$ although it does not represent any spectral sensitivity of photoreceptors on the retina. The intensity of the lighting energy used in this experiment was too low to damage the retina but enough to cause eye fatigue. In this study, we compared the relative intensities of light as blue-light hazard stimuli among the three SPD conditions. However, the rank order of the SPD conditions was V-LED>S-LED>B-LED. The rank order of the SPDs does not match our experimental results. Therefore, we concluded that it was inappropriate to evaluate the effect of SPD on eye fatigue using the blue-light hazard function.

Next, we used the spectral sensitivity of the S-cone $(S(\lambda))$, with a peak sensitivity at a shorter wavelength than the relative luminous efficiency function $(V(\lambda))$. Rea et al. proposed a brightness perception model that combines the spectral sensitivity functions of $S(\lambda)$ and $V(\lambda)^{26)}$. 


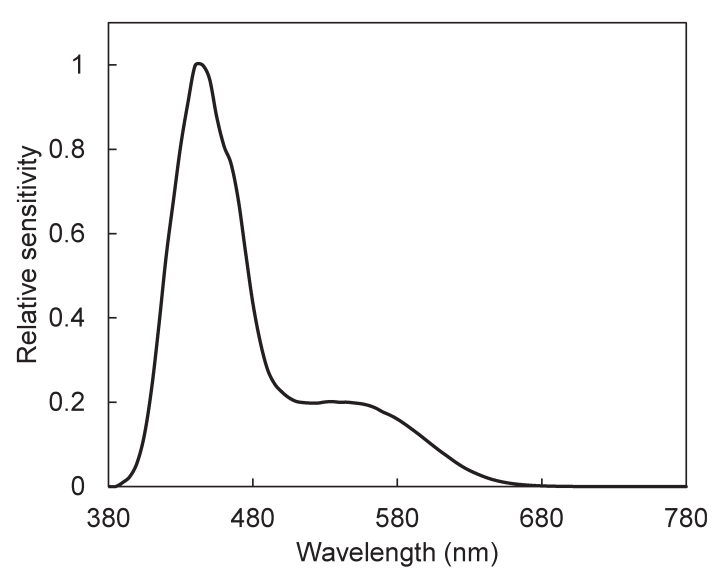

Figure 13 Spectral sensitivity function $(B(\lambda))$ proposed by Rea et al. $B(\lambda)=V(\lambda)+5 \times S(\lambda)$ where $V(\lambda)$ is the relative luminous efficiency function and $S(\lambda)$ is.

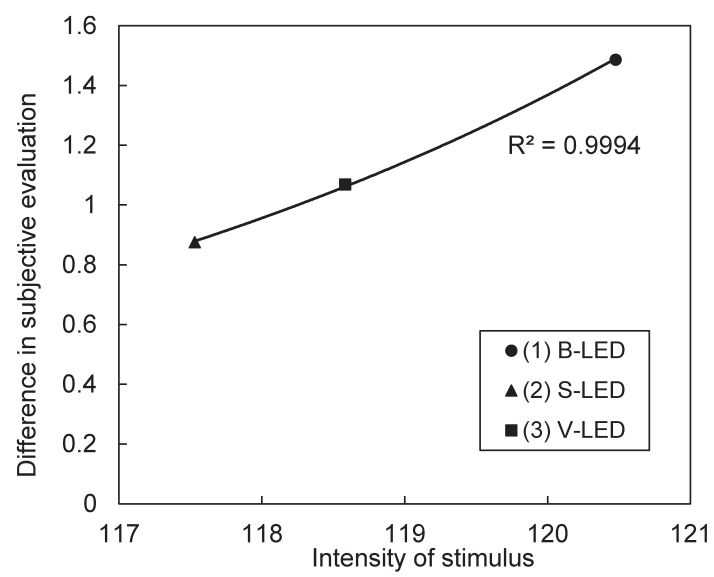

Figure 14 Relationship between the intensity of the stimuli and the difference in subjective eye-fatigue evaluation for Statement (5): I have eye fatigue.

In this brightness perception model, the contribution rate of $S(\lambda)$ to the brightness perception increases as the illuminance of the visual target increases. According to the brightness perception model $(B(\lambda))$, we determined the contribution rate of $S(\lambda)$ as five. Figure 13 shows the combined spectral sensitivity function by using the brightness perception model. The function in the figure is $B(\lambda)=V(\lambda)+5 \times S(\lambda)$. Using the spectral sensitivity function, we calculated the intensity of the stimuli for the three light sources. We examined the correspondence between the intensities of the stimuli regarding brightness perception and subjective eye-fatigue (Statement (5): I have eye fatigue.) evaluation values. Figure 14 shows the relationship between the intensities of the stimuli calculated using the brightness perception model and subjective eye-fatigue evaluations. Figure 14 suggests that the larger the stimulus intensity, the larger the increase in subjective eye fatigue. The coefficient of determination (R-square) of the regression curve was high at 0.999 .
Therefore, we conclude that eye fatigue increased as the stimulus intensity calculated by the brightness perception model increased, which is true only when visual targets are illuminated at high illuminance so that people can clearly see the targets. However, attention must be paid to the fact that the differences among the three LED sources in the horizontal axis were very small in Figure 14.

Zhang et al. discussed the possibility of the contribution of the intrinsically photoreceptive retinal ganglion cell (ipRGCs) to eye fatigue ${ }^{2)}$. According to their discussion, we tried to apply the ipRGC's spectral sensitivity function to the SPDs of the three light sources. After calculating the stimulation intensity of the three light sources using the spectral characteristics of melanopsin, we found the order was B-LED (blue-light excited LED) $<$ S-LED (solar LED) $<$ V-LED (violet-light excited LED). The order of the results was the reverse of the order of eye fatigue obtained in our experiment. Therefore, we considered that ipRGC might little contribute to eye fatigue. Further research is needed to clarify the photoreceptors related to eye fatigue.

\section{Conclusion}

We investigated the effect of blue light emitted by phosphor-converted white LEDs on people's eye fatigue by conducting an experiment in which participants performed a visual task under lighting conditions using a white LED in which blue light excites phosphors and two types of white LEDs excited by violet light. One of the two violet-excited LEDs had a SPD mimicking that of the sun. During the experiment, we measured participants' subjective eye fatigue, number of eyeblinks, accommodation power and $\mathrm{CFF}$ as the dependent variables.

The results of the subjective eye-fatigue evaluation show that participants' eye fatigue under the blueexcited LED lighting was worse than under the solar LED or the violet-excited LED.

In the range of the white LEDs used in our experiment, participants' eye fatigue under lighting conditions with blue-excited white LEDs might be worse than under violet-excited white LEDs. The results of the objective response measurements show that the fatigue of the eye muscles (extraocular and ciliary muscles) are the principal factor causing subjective eye fatigue.

Furthermore, we investigated the mechanisms of the effect of the light SPD on eye fatigue. In the analysis, we quantified the intensity of the stimulus using a brightness perception model that combined the spectral sensitivity of the S-cone and spectral sensitivity of the cone ${ }^{26)}$. Consequently, the higher the stimulus of brightness perception, the higher the eye fatigue. However, attention must be paid to the fact that the differences in stimulus intensity among the three LED sources were 
very small. Therefore, further research on the mechanisms of eye fatigue is needed.

\section{References}

(1) Wang, Y., Zhong, X., Zhang, Y., Tu, Y., Wang, L., Chen, Y., Zhang, C. and Zhou, W.: Visual fatigue following long-term visual display terminal work under different light sources, Light. Res. Technol., 49-8, pp. 1034-1051 (2017).

(2) Zhang, Y., Tu, Y., Wang, L. and Zhang, W.: Assessment of visual fatigue under LED tunable white light with different blue components, J. Soc. Inf. Disp., 28-1, pp. 24-35 (2020).

(3) Mochizuki, E. and Kimura, H.: Effects of difference of spectral power distribution on visual fatigueComparison between visual fatigue caused by VDT works under LEDs and that under fluorescent lamps-, Transactions of AIJ, J. Environ. Eng., 75-647, pp. 35-41 (2010).

(4) Kubo, C., Yabuta, Y., Yamaba, K. and Ayama, M.: Effect of spectral radiant distribution of lighting on visual fatigue, J. Sci. Technol. Light., 98, pp. 79-86 (2014).

(5) Chen, Y. L., Zhang, Y. H., Jia, L., Yong, Y., Yang, Y. B. and $\mathrm{Xu}, \mathrm{P}$.: Evaluate fatigue of blue light influence under general LCD, Nice blue LCD displays, Sid Symp. Dig. Tec. Papers, 48, pp.1367-1370 (2017).

(6) Chiu, H. P. and Liu, C. H.: The effects of three blue light filter conditions for smartphones on visual fatigue and visual performance, Hum. Factors Ergon. Manuf. Serv. Ind., 30-1, pp. 83-90 (2020).

(7) Nishimura, T., Morimoto, K., Kishimoto, T. and Nii, M.: Correlation between subjective and objective rating of fatigue in VDT operations, J. Inst. Televis. Eng. Jpn., 40, pp. 1239-1244 (1986).

(8) Gang, G. L. and Sakamoto, K.: Studies on the evaluation of visual fatigue in VDT work by involuntary eye movement (flick), The Japanese Journal of Ergonomics, 32-2, pp. 87-97 (1996).

(9) Kaneko, K. and Sakamoto, K.: Spontaneous blinks as a criterion of visual fatigue during prolonged work on visual display terminals, Percept. Mot. Skills, 92-1, pp. 234-250 (2001).

(10) Kimura, T., Hayasaka, Y., Segawa, N., Yamazaki, K., Murayama, Y. and Miyazaki, M.: A proposal on an evaluation method towards the development of a human interface with less visual fatigue, Information Processing Society of Japan, 44, pp. 2587-2597 (2003).

(11) Kondo, Y., Nishimura, Y., Ishii, H., Shimoda, H. and Yoshikawa, H.: A study on an objective examination method of eye strain by using Eye-Sensing Display, Human Interface: Proceedings of the Human Interface Symposium, 2, pp. 643-648 (2006).
(12) Lin, C. J., Feng, W. Y., Chao, C. J. and Tseng, F. Y.: Effects of VDT workstation lighting conditions on operator visual workload, Ind. Health, 46-2, pp. 105-111 (2008).

(13) Chiang, H. C., Lee, D. G., Ko, B. W., Koga, T., Hirate, K., Munakata, J. and Yoshizawa, N.: A Study on the psychological and physiological effects by LED lightings for workspace, Transactions of AIJ, J. Environ. Eng., 75-654, pp. 683-690 (2010).

(14) Bang, J. W., Heo, H., Choi, J. S. and Park, K. R.: Assessment of eye fatigue caused by 3D displays based on multimodal measurements, Sensors (Basel), 14-9, pp. 16467-16485 (2014).

(15) Hashimoto, K.: Measurement of general fatigue, The Japanese Journal of Ergonomics, 17-3, pp. 107-113 (1981).

(16) Tada, H., Yamada, H. and Fukuda, K.: Psychology of blinking. Japan. Kitaooji Shobo Publishing, pp.158-168 (1991).

(17) Kajita, M.: The role of accommodative function in refractive correction-Aspects of asthenopia that could be understood through medical treatment-, Japanese Journal of Visual Science, 33, pp. 138-146 (2012).

(18) Japanese Society of Occupational Health: How to use "Jikaku-syo shirabe (survey on subjective symptoms).” Retrieved 13 August 2020, from http://square.umin.ac.jp/of/

(19) Nishimura, T. and Morimoto, K.: Readability of Japanese characters on CRT and visual fatigue, J. Inst. Televis. Eng. Jpn., 37, pp. 730-735 (1983).

(20) Takeda, M., Hayashi, Y. and Suzuki, K.: Differences in CFF value obtained from limits method and the adjustment method and, skilled and unskilled subjects, The Japanese Journal of Ergonomics, 31-4, pp. 295-303 (1995).

(21) Winn, B., Pugh, J. R., Gilmartin, B. and Owens, H.: The frequency characteristics of accommodative microfluctuations for central and peripheral zones of the human crystalline lens, Vision Res., 30-7, pp. 1093-1099 (1990).

(22) Jeng, W. D., Ouyang, Y., Huang, T. W., Duann, J. R., Chiou, J. C., Tang, Y. S. and Mang, O. Y.: Research of accommodative microfluctuations caused by visual fatigue based on liquid crystal and laser displays, Appl. Opt., 53-29, pp. 76-84 (2014).

(23) Kajita, M., Muraoka, T. and Orsborn, G.: Changes in accommodative micro-fluctuations after wearing contact lenses of different optical designs, Cont. Lens Anterior Eye, 43-5, pp. 493-496 (2020).

(24) International Electrotechnical Commission: IEC 62471 Photobiological Safety of Lamps and Lamp Systems, Geneva: IEC, (2006).

(25) International Electrotechnical Commission: IEC 62 778 Application of IEC 62471 for the Assessment 
of Blue Light Hazard to light Sources and luminaires, Geneva: IEC, (2014).

(26) Rea, M. S., Radetsky, L. C. and Bullough, J. D.: Toward a model of outdoor lighting scene brightness, Light. Res. Technol., 43-1, pp. 7-30 (2011). 